\title{
Diabetes mellitus
}

\section{Analoginsuline bieten klinische und praktische Vorteile}

— In Deutschland werden Analoginsuline viel seltener verordnet als in Schweden, Frankreich, Großbritannien oder Italien, obwohl Analoginsuline hierzulande aufgrund von Rabattverträgen mit den Krankenkassen für Patienten mit Typ-2-Diabetes das gleiche kosten wie Humaninsuline, während sie in den genannten Ländern deutlich teurer sind. Wie Dr. Rolf Renner, Göppingen, und Dr. Rainer Lundershausen, Erfurt, im Rahmen eines von Novo Nordisk unterstützten Expertenworkshops deutlich machten, gibt es zahlreiche Argumente, die für die Verwendung moderner Analoginsuline bei Typ-2-Diabetikern sprechen.

\section{Unkomplizierte, komfortable Therapie}

„Wichtig ist zunächst, dass die Blutzuckereinstellung unter Verwendung von Kurzund Langzeitinsulinanaloga bei gleichzeitiger Verringerung der Hypoglykämiefrequenz deutlich verbessert werden kann", informierte Renner. Da sich subkutan applizierte Insulinanaloga in ihrem pharmakokinetischen Profil eher dem endogenen phy-

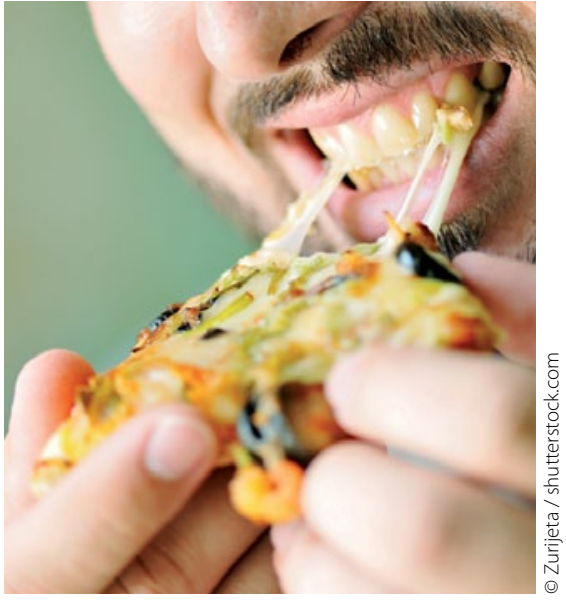

Essen wie ein Gesunder - für viele Diabetiker ein Traum.

siologischen Insulinprofil annähern als Humaninsulin, müsse beim Einsatz von Analoginsulinen außerdem in der Regel kein Spritz-Ess-Abstand eingehalten werden.

\section{Zwischenmahlzeiten unnötig}

„Im Rahmen einer intensivierten Insulintherapie mit kurz- und lang wirksamen In- sulinanaloga sind auch keine Zwischenmahlzeiten mehr erforderlich. Das ist vor allem deshalb von großer Bedeutung, weil Zwischenmahlzeiten die Fettvermehrung begünstigen und die Diabeteseinstellung verschlechtern", betonte Renner.

Nach Lundershausen sind unter Analoginsulinen keine Sicherheitsrisiken zu erkennen. „Weder ist das Karzinomrisiko unter lang wirksamen Insulinanaloga erhöht - dies belegen klinische Studien und die Beobachtungen aus dem bereits 15-jährigen Einsatz in der Praxis - noch gibt es hinsichtlich des kardiovaskulären Risikos Bedenken". Es sei vielmehr vorteilhaft, dass sich die als kardiovaskuläre Risikofaktoren bekannten postprandialen Blutzuckerspitzen beispielsweise mit Insulin aspart (Novo Rapid $^{\circledR}$ ) stärker und schneller senken lassen als mit Humaninsulin (Rys $P$ et al., Diabetes Metab 2011;37:190-200).

- Dr. Silke Wedekind

Quellen: Springer Medizin Expertenworkshop "Moderne Insulintherapie aus Sicht der Experten", München, März 2013 (Veranstalter: Novo Nordisk)

\section{Ab jetzt für den SilverStar 2013 bewerben!}

- Insgesamt 55 Initiativen, Selbsthilfegruppen, Kliniken und Praxen hatten ihre Projekte für den SilverStar Förderpreis 2012 eingereicht.

Der Preis wird dieses Jahr das dritte Mal ausgelobt. Er soll Bewerber und Projekte unterstützen, die sich die Betreuung von älteren Diabetikern zur Aufgabe gemacht haben.

Ob Ärzte, Pflegekräfte bzw. Apotheker, oder engagierte Angehörige, Freunde, Nachbarn bzw. Betroffene: Jede Einzelperson, Gruppe oder Organisation kann sich bis zum 30. Juni 2013 um den SilverStar bewerben.

Nähere Infos unter: www.silverstar-preis.de Berlin-Chemie
Ginkgo-Präparate im Öko-Test

- Das Magazin "Öko-Test" hat zehn Ginkgo-Präparate zur Behandlung bei Gedächtnisstörungen getestet [„Mittel gegen Gedächtnisstörungen/ Stärkungsmittel. Vergiss es!", Öko-TestMagazin 2013;4:50-57]. Nur eines, Tebonin ${ }^{\circledR}$ konzent 240 mg, bekam die Note "gut".

Von den neun anderen untersuchten Ginkgoprodukten erhielt eines die Note "befriedigend", alle übrigen nur ein "ausreichend" oder gar "mangelhaft". Laut Testbericht kam es in der pharmakologischen Begutachtung darauf an, ob sich ein Arzneimittel in gut durchgeführten Studien für die beanspruchten Anwendungsgebiete als wirksam erwiesen habe.
Mit Ausnahme von Tebonin ${ }^{\circledast}$ lautet das Fazit des Magazins, dass die Wirksamkeit der Ginko-Präparate wegen der widersprüchlichen Studienlage nicht richtig belegt werden könne. Ausschlaggebend für seine positive Bewertung von EGb 761® nannte "ÖkoTest" das Urteil des Instituts für Qualität und Wirtschaftlichkeit im Gesundheitswesen (IQWiG) von 2008 [Ginkgo-haltige Präparate bei Alzheimer Demenz. Abschlussbericht A05-19B. IQWiG, 2008]. Das IQWiG erkannte nach umfangreicher Studienauswertung einen Nutzen für das Therapieziel „Aktivitäten des täglichen Lebens" an, wenn Patienten mit Alzheimer-Demenz täglich eine Dosis von 240 mg EGb 761 ${ }^{\circledR}$ einnehmen.

Dr. Willmar Schwabe 